\title{
Correlation between 1-FABP, Blood Routine and Grading of Necrotising Enterocolitis
}

\author{
Wei Su${ }^{1}$, Yanjun $\mathrm{Li}^{2}$, Yuebin Wang ${ }^{1}$, Lixiang $\mathrm{Li}^{1}$ and Jianhui Yan ${ }^{1}$ \\ ${ }^{1}$ Department of Paediatrics, Affiliated Hospital of Xiangnan University (Clinical College), China \\ ${ }^{2}$ Department of Orthopedic Trauma, Zhuzhou Hospital Affiliated to Xiangya School of Medicine, Central South University, China
}

\begin{abstract}
Correlation was sought between serum intestinal fatty acid binding protein (I-FABP), blood routine examination indexes, and necrotizing enterocolitis (NEC) disease classification. According to Bell-NEC grade, 86 children with NEC were divided into mild group (46 cases) and severe group (40 cases). Serum I-FBAP and blood routine indices including white blood cells (WBC), platelets (PLT), neutrophils and lymphocytes were determined. Serum levels of I-FABP and WBC in severe group were higher than those in mild group (both $p<0.001$ ), and serum level of PLT was lower than that in mild group $(p<0.001)$. NEC grade was positively correlated with I-FABP and WBC $(r=0.930, p<0.001 ; r=0.946, p$ $<0.001$ ), negatively correlated with PLT $(r=-0.602, p<0.001)$, and had weak correlation with neutrophils and lymphocytes $(r=0.186, p=0$. 087; $r=0.072, p=0.509$ ). In conclusion, serum levels of I-FABP, WBC and PLT were correlated with the severity of NEC in children, which could be used as a reference index to judge prognosis of NEC children.
\end{abstract}

Key Words: Necrotizing enterocolitis of newborn (NEC), Serum, Intestinal fatty acid binding protein (I-FABP), Routine blood indices.

How to cite this article: Su W, Li Y, Wang Y, Li L, Yan J. Correlation between 1-FABP, Blood Routine and Grading of Necrotising Enterocolitis. J Coll Physicians Surg Pak 2021; 31(02):238-239.

Necrotising enterocolitis (NEC) is a serious life-threatening disease in the neonatal stage. Clinically, abdominal distension, vomiting, hematochezia and severe shock are the main clinical manifestations. Abdominal X-ray examination is characterised by cystic gas accumulation in intestinal wall.

Children with mild NEC have a good prognosis, while children with severe NEC may die, if they are not treated in time. Therefore, early diagnosis of NEC is very important for the treatment and prognosis. The onset of NEC may be related to intestinal mucosal ischemia and hypoxia, and relative reduction of blood supply or bacterial infection in children. ${ }^{1}$ However, there is a lack of effective and feasible diagnostic methods for the reversible period of intestinal mucosal ischemia, which makes it difficult for NEC children to be treated in time.

At present, theearly diagnosis ofNECismainly based on clinical manifestations and imaging results; but sometimes it lacks specificity, such as non-specific manifestations, unstable body temperature, feeding intolerance, vomiting, abdominal distension, hematochezia, and low blood perfusion pressure, which are sometimes difficult to distinguish from intestinal obstruction induced by gastrointestinal peristalsis disorder, electrolyte disorder or septicemia of premature infants. ${ }^{2}$

Correspondence to: Dr. Jianhui Yan, Department of Paediatrics, The Affiliated Hospital of Xiangnan University (Clinical College), China

E-mail: bjzra5@163.com

Received: May 14, 2020; Revised: September 24, 2020;

Accepted: November 24, 2020

DOI: https://doi.org/10.29271/jcpsp.2021.02.238
Although intestinal loop dilatation, intestinal wall gas accumulation, and portal vein gas accumulation are specific changes of NEC on abdominalX-ray plain films, they are often atypical in the early stage.

White blood cells (WBC), platelets (PLT) and other indicators are clinical blood routine examination indicators, which can reflect the pathogenesis of systemic infection in NEC children, but it is difficult to accurately reflect the degree of intestinal epithelial cell injury and necrosis. ${ }^{3}$ Intestinal fatty acid binding protein (I-FABP) is a water-solubleprotein secreted by small intestinal monolayercolumnarepithelial cells and has good organ specificity. It only exists in gastrointestinal mucosa, mainly distributed in jejunum and ileum, with the most obvious accumulation at the top of jejunal mucosa microvilli. ${ }^{4}$ Under normal circumstances, there was only a small amount of IFABP in blood circulation. When intestinal ischemia occurs, intestinal mucosal villi were affected first, and the permeability of mucosal cell membrane increases. I-FABP can quickly enter blood circulation through cell membrane, capillaries, lymphatic capillaries and portal vein. Blood I-FABP level can reflect intestinal damage at an early stage. Some studies reavealed that I-FABP played a certain role in the early diagnosis and guiding treatment of NEC children. ${ }^{5,6}$ At present, the correlation between I-FABP, blood routine and necrotising enterocolitis disease grade is still unclear.

The purpose of this study was to explore the correlation between serum I-FABP, blood routine examination indexes and NEC disease classification.

After approval by the Hospital Ethics Committee, this experimental study was carried out in the Affiliated Hospital of Xiangnan University (ClinicalCollege), China, from April2018to April2020. Eighty-sixchildren with NEC were selected as the research subjects. According to Bell-NEC grade, they were divided into mild group (46 cases) and severe group (40 cases). The inclusion criteria were in line with NEC diagnostic criteria. Children who were finally diagnosed as non-NEC, 
complicated with other serious infections, with obvious congenital malformation or metabolic diseases at birth, were excluded. According to Bell-NEC classification, children in Stage I and Stage Ila were divided into mild group (46 cases), and children in Stage IIb and Stagelll were divided into severe group ( 40 cases). Five $\mathrm{mL}$ of venous blood beforetreatment wastaken forl-FABPand blood routine examination. Blood routine indexes included white blood cells (WBC), platelets (PLT), neutrophils and lymphocytes. Serum I-FABP was detected by enzyme-linked immunosorbentassay.

SPSS 25.0 statistical software was used to process the data. ShapiroWilk test was used to check normality of quantitative variables. The quantitative data conforming to normal distribution were expressed by mean \pm standard deviation, and the independent sample t-test was used for the comparison of two pairs. Spearman grade correlation was used to analyse the correlation between I-FABP, blood routine examination indexes, and NEC children's disease grade.

Table I: Comparison of serum I-FABP and blood routine of two groups of children.

\begin{tabular}{|c|c|c|c|}
\hline Index & Mild group $(n=46)$ & Severe group $(n=40)$ & p-value \\
\hline I-FABP $(\mu \mathrm{g} / \mathrm{L})$ & $25.72 \pm 1.77$ & $31.70 \pm 2.26$ & $<0.001$ \\
\hline WBC $\left(\times 10^{9} / \mathrm{L}\right)$ & $7.68 \pm 0.53$ & $20.37 \pm 1.45$ & $<0.001$ \\
\hline PLT $\left(\times 10^{9} / \mathrm{L}\right)$ & $194.85 \pm 13.43$ & $100.09 \pm 7.13$ & $<0.001$ \\
\hline Neutrophils (\%) & $0.65 \pm 0.05$ & $0.64 \pm 0.04$ & 0.154 \\
\hline Lymphocytes (\%) & $0.27 \pm 0.02$ & $0.28 \pm 0.03$ & 0.080 \\
\hline
\end{tabular}

Among 86 NEC children, 50 were males (58.14\%) and 36 were females $(41.86 \%)$. The mean gestational age was (35.18 \pm 2.93$)$ weeks; birth weight 800-4 660 g, mean (2 098. $55 \pm 214.62)$ g; BellNEC graded 20 cases (23.26\%) in Stage l, 26 cases (30.23\%) in Stage Ila, 23 cases (26.74\%) in Stage Ilb, and 17 cases (19.77\%) in Stage III. The $p<0.05$ was statistically significant.

The levels of I-FABP and WBC in severe group were significantly higher than those in mild group (both $p<0.001$, Table I), and the levels of PLT was significantly lower than those in mild group ( $p$ $<0.001$, Table I). There was no significant difference in the levels of neutrophils and lymphocytes between the two groups $(p=0.154$, 0.080 , Tablel).

Spearman grade correlation analysis showed that NEC grade was positively correlated with I-FABP and WBC $(r=0.930, p<0.001$; and $r=0.946, p<0.001$, respectively), negatively correlated with PLT ( $r=-0.602, p<0.001)$, and had weak correlation with neutrophils and lymphocytes $(r=0.186, p=0.087$; and $r=0.072, p=0.509$, respectively).

At present, there was no effective and reliable index to predict the disease condition of NEC children. Blood routine examination is a common clinical examination index. In this study, the levels of I-FABP and WBC in the severe group were significantly higher than those in the mild group, and the levels of PLT were significantly lower than those in the mild group.

There was no significant difference in the levels of neutrophils and lymphocytes between the two groups. Children with NEC, whose levels of I-FABP, WBC and PLT showed obvious abnormal changes in a short period of time, may have a higher risk of developing into severe diseases. From the correlation analysis, NEC grade was positively correlated with I-FABP and WBC, negatively correlated with PLT, and weak correlation with neutrophils and lymphocytes. It showed that the levels of I-FABP, WBC and PLT are correlated with the severity of NEC in children, which could be used as a reference index to judge the prognosis of NEC children. The limitation of this study lies in the small number of research samples included, which needs to be further studied in the future.

\section{PATIENTS' CONSENT:}

Informed consents were obtained from all the patients' family members.

\section{CONFLICT OF INTEREST:}

Authors declared no conflict of interest.

\section{AUTHORS' CONTRIBUTION:}

WS: Conception or design of the work; drafting the work.

YL, YW, LL: Revising it critically for important intellectual content. JY: Revising it critically for important intellectual content; final approval of the version to be published.

\section{REFERENCES}

1. Walker WA, Meng D. Breast milk and microbiota in the premature gut: A method of preventing necrotizing enterocolitis. Nestle Nutr Inst Workshop Ser 2020; 94: 103-12. doi: 10.1159/000505337.

2. Martynov I, Raedecke J, Klima-Frysch J, Kluwe W, Schoenberger J. The outcome of Bishop-Koop procedure compared to divided stoma in neonates with meconium ileus, congenital intestinal atresia and necrotizing enterocolitis. Medicine (Baltimore) 2019; 98(27):e16304. doi: 10.1097/MD.0000000000016304.

3. Yu M, Liu G, Feng Z, Huang L. Combination of plasma white blood cell count, platelet count and C-reactive protein level for identifying surgical necrotizing enterocolitis in preterm infants without pneumoperitoneum. Pediatr Surg Int 2018; 34(9):945-50. doi: 10.1007/s00383-018-4305-6.

4. Plat VD, Derikx JPM, Jongen AC, Nielsen K, Sonneveld DJA, Tersteeg JJC, et al. Diagnostic accuracy of urinary intestinal fatty acid binding protein in detecting colorectal anastomotic leakage. Tech Coloproctol 2020; 24(5): 449-54. doi: 10. 1007/s10151-020-02163-3.

5. Yang $G$, Wang $Y$, Jiang $X$. Diagnostic value of intestinal fattyacid-binding protein in necrotizing enterocolitis: A systematic review and meta-analysis. Indian J Pediatr 2016; 83 (12-13):1410-9. doi: 10.1007/s12098-016-2144-9.

6. Schurink M, Kooi EM, Hulzebos CV, Kox RG, Groen H, Heineman $E$, et al. Intestinal fatty acid-binding protein as a diagnostic marker for complicated and uncomplicated necrotizing enterocolitis: A prospective cohort study. PLoS One 2015; 10(3):e0121336. doi: 10.1371/journal.pone. 0121336 . 